\title{
ON A THEOREM OF GROMOV AND MILMAN
}

\author{
TADEUSZ JANUSZKIEWICZ
}

\begin{abstract}
We give simple examples of topologically trivial bundles which admit no uniformly continuous section.
\end{abstract}

In a recent paper [2] Gromov and Milman obtained the following result: There exists a bundle on some noncompact metric space, which is topologically trivial, but which admits no uniformly continuous section. The proof makes use of some very interesting ideas, which are, however, not very standard. Our aim here is to give much simpler examples, using some more standard tools.

Recall from [3] that with the uniform structure defined by a Riemannian metric $g$ on a manifold $M$, one associates the de Rham complex of differential forms bounded with respect to $g$ and its homology $H_{b}^{*}(M, g)$. The Chern-Weil theory implies that characteristic forms define comhomology classes in $H_{b}^{*}(M, g)$ depending only on the uniform structure of $g$. In particular, one has the Euler class.

Now with $M, g$ one associates the unit sphere bundle $T_{1}(M)$ carrying the obvious uniform structure on its total space. The following proposition is well known.

Proposition. If $M$ is noncompact, then $T_{1}(M)$ admits a continuous section.

This is proved by "pushing singularities to infinity". In contrast one has the following

THEOREM. If the Euler class is nonzero in $H_{b}^{*}(M, g)$ then $T_{1}(M)$ admits no uniformly continuous section.

Proof. By work of Chern [1] one has the form $\eta$ living on the total space of $T_{1}(M)$ such that $d \eta$ is the lift of the Euler class to $T_{1}(M)$. Moreover, $\eta$ is locally computable in terms of the metric and, thus, is bounded with respect to the metric if it is $C^{2}$. Now if there is a uniformly continuous section of $T_{1}(M)$, we can approximate it by a smooth section with uniformly bounded derivatives, say $\alpha$. Then the pullback $\alpha^{*} \eta$ is a form on $M$ bounded with respect to the metric. Since

$$
d \alpha^{*} \eta=\alpha^{*} d \eta=\alpha^{*} \pi^{*} e u=e u,
$$

the Euler form vanishes in $H_{b}^{n}(M, g)$, contradicting the hypothesis.

Received by the editors March 26, 1984.

1980 Mathematics Subject Classification. Primary 53C20, 57R20, 55R25.

Key words and phrases. Uniform structures, Chern-Weil theory. 
COROLlaRY. The unit sphere bundle of a surface with constant negative curvature and finite volume admits no uniformly continuous section.

For other examples see [3].

\section{BIBLIOGRAPHY}

1. S. S. Chern, A simple intrinsic proof of the Gauss-Bonnet formula for closed Riemannian manifolds, Ann. of Math. (2) 45 (1944), 747-752.

2. M. Gromov and V. D. Milman, The topological application of the isoperimetric inequality, Amer. J. Math. 105 (1983), 843-854.

3. T. Januszkiewicz, Characteristic invariants of noncompact Riemannian manifolds, Topology (to appear).

Instytut Matematyczny UWr, Pl. GrunWaldzki 2/4, 50 - 384 Wroclaw, Poland 